\title{
Existence of Crystal Bases for Kirillov-Reshetikhin Modules of Type $D$
}

\author{
Dedicated to Professor Noriaki Kawanaka on his sixtieth birthday
}

By

Masato OKADO*

\begin{abstract}
We show that a crystal base exists for any Kirillov-Reshetikhin module of type $D_{n}^{(1)}$, generalizing the result of Kang et al. for the end nodes of the Dynkin diagram of $D_{n}$.
\end{abstract}

\section{$\S 1 . \quad$ Introduction}

Let $\mathfrak{g}$ be an affine algebra and let $U_{q}^{\prime}(\mathfrak{g})$ be the corresponding quantum affine algebra without degree operator. Among irreducible finite-dimensional $U_{q}^{\prime}(\mathfrak{g})$-modules there exists a distinguished family called Kirillov-Reshetikhin modules (KR modules for short). They were introduced in [17] in connection with a certain conjectural formula of multiplicities of irreducible $U_{q}\left(\mathfrak{g}_{0}\right)$ modules in a tensor product of those modules. Here $\mathfrak{g}_{0}$ stands for the finitedimensional simple Lie algebra whose Dynkin diagram is obtained by removing the 0 -vertex, that is prescribed in [10], from that of $\mathfrak{g}$. It is known [5, 4] that irreducible finite-dimensional $U_{q}^{\prime}(\mathfrak{g})$-modules are classified by $n$-tuples of polynomials called Drinfeld polynomials, where $n$ is the rank of $\mathfrak{g}_{0}$.

Let us define KR modules by the Drinfeld polynomials. Let $k \in\{1,2, \ldots$, $n\}, l \in \mathbb{Z}_{>0}$ and $a$ an invertible element of $\mathbb{Q}(q)$. A KR module $\tilde{W}_{l, a}^{(k)}$ is defined to be the unique irreducible finite-dimensional $U_{q}^{\prime}(\mathfrak{g})$-module that has

$$
P_{i}(u)= \begin{cases}\left(1-a q_{i}^{1-l} u\right)\left(1-a q_{i}^{3-l} u\right) \cdots\left(1-a q_{i}^{l-1} u\right) & \text { if } i=k, \\ 1 & \text { otherwise }\end{cases}
$$

Communicated by M. Kashiwara. Received November 17, 2006.

2000 Mathematics Subject Classification(s): 17B37.

*Department of Mathematical Science, Graduate School of Engineering Science, Osaka University, Toyonaka, Osaka 560-8531, Japan. 
as Drinfeld polynomials. (See Section 2.1 for the definition of $q_{i}$.) When $l=1$ it is also called a fundamental representation. Since a fundamental representation is known to have a crystal base [14], we choose $a^{\dagger}$ so that $\tilde{W}_{l, a^{\dagger}}^{(k)}$ has a crystal base and redefine $W_{l, a}^{(k)}=\tilde{W}_{l, a^{\dagger} a}^{(k)}$. $W_{l, 1}^{(k)}$ is also denoted simply by $W_{l}^{(k)}$.

The above-mentioned conjectural multiplicity formula was shown when $\mathfrak{g}$ is non-twisted by combining two results. The first one is the proof of certain algebraic relations among characters of KR modules, called $Q$-systems, presented in [20] for simply-laced cases and in [8] for all non-twisted cases. The second one is a derivation of the multiplicity formula, called fermionic formula, in [16] for type $A$ and in [7] for all non-twisted cases, by using the $Q$-systems. However, it deserves to emphasize that there is also a $q$-analogue of the conjecture, called $X=M$ conjecture [7,6]. The definition of $X$ requires the existence of the crystal base of a KR module. Despite many efforts as in $[12,11,25,18,9,14,19,23,2]$, this existence problem is yet to be settled. For type $D$ for instance, the crystal base has been shown to exist for $W_{l}^{(k)}$ where $k=1, n-1, n ; l \in \mathbb{Z}_{>0}$ in $[12]$ and for $W_{1}^{(k)}$ for arbitrary $k$ in $[18,14]$.

In this paper we prove the following theorem, thereby settling the problem for type $D$.

Theorem 1.1. For $2 \leq k \leq n-2$ and $l \geq 1$, the $U_{q}^{\prime}\left(D_{n}^{(1)}\right)$-module $W_{l}^{(k)}$ has a crystal pseudobase.

Here $(L, B)$ is said to be a crystal pseudobase if $(L, B /\{ \pm 1\})$ is a crystal base. (See Definition 2.1 for the definition of a crystal base.) Let us give a short sketch of our proof. We follow the technique already developed in [12], namely, from a fundamental representation $W_{1}^{(k)}$ we construct $W_{l}^{(k)}$ for any $l$ by fusion construction (Section 2.3), and we apply a criterion of the existence of a crystal pseudobase (Proposition 2.6) to the constructed module $W_{l}^{(k)}$. Practically, we need to check two conditions $((2.27)$ and (2.28)). Checking the second one is not difficult, if once we find out the vectors $\left\{u_{j}\right\}$ correctly, whereas checking the first one requires information on the image $W$ and the kernel $N$ of the $R$-matrix $R(x, y): W_{1, x}^{(k)} \otimes W_{1, y}^{(k)} \longrightarrow W_{1, y}^{(k)} \otimes W_{1, x}^{(k)}$ at $x / y=q^{2}$. Up to now such information was obtained by calculating the spectral decomposition of the $R$-matrix when dealing with $W_{l}^{(k)}$ for higher $l$. It seems to be the reason why showing the existence of crystal bases of $W_{l}^{(k)}$ for higher $k$ has not been succeeded so far, since the calculation of the corresponding $R$-matrix is too much complicated. However, thanks to the result by Nakajima [20], we are now able to identify $W$ and $N$ with tensor products of KR modules (Lemma 3.4). Using the crystal base of $W$ and a property of a bilinear form between 
$W_{1, q}^{(k)} \otimes W_{1, q^{-1}}^{(k)}$ and $W_{1, q^{-1}}^{(k)} \otimes W_{1, q}^{(k)}$, we can check the first condition of the criterion. It is known that a $U_{q}(\mathfrak{g})$-module with a connected crystal base is irreducible. Therefore, once $W_{l}^{(k)}$ is shown to have a crystal pseudobase, it follows that $W_{l}^{(k)}$ is an irreducible finite-dimensional module with the desired Drinfeld polynomials, since it is a simple quotient of $W_{1, q^{1-l}}^{(k)} \otimes W_{1, q^{3-l}}^{(k)} \otimes \cdots \otimes$ $W_{1, q^{l-1}}^{(k)}$.

After the author finished the manuscript, he learned from Kashiwara that the module $W_{l}^{(k)}$ can be shown to be irreducible by Theorem 9.2 of [14]. Once it is established, the character is known by [3]. Hence it turns out that there is no need to prove the inequality of the character in (i) just after Proposition 3.7. However, this does not seem to prove that $W_{l}^{(k)}$ is isomorphic to a module of the form of $V^{\otimes l} / \sum_{i=0}^{l-2} V^{\otimes i} \otimes N \otimes V^{\otimes(l-2-i)}$. The author was also informed from Nakajima that the existence of a polarization on the fundamental representation $W_{1}^{(k)}$ was shown in [24] (see also [21, 1] for more general results). Hence similar calculations of the prepolarization as in Section 5 will give a proof of the existence of crystal bases for KR modules of other quantum affine algebras.

\section{§2. Crystal Base and Fusion Construction}

\section{$\S 2.1$. Crystal base}

In this subsection we briefly recall the definition of crystal bases. For more details along with the definition of $U_{q}(\mathfrak{g})$, refer to [13].

Let $\mathfrak{g}$ be a symmetrizable Kac-Moody Lie algebra and let $M$ be a $U_{q}(\mathfrak{g})$ module. $M$ is said to be integrable if $M=\oplus_{\lambda \in P} M_{\lambda}$, $\operatorname{dim} M_{\lambda}<\infty$ for any $\lambda$, and for any $i, M$ is a union of finite-dimensional $U_{q}\left(\mathfrak{g}_{i}\right)$-modules. Here $P$ is the weight lattice of $\mathfrak{g}, M_{\lambda}$ is the weight space of $M$ of weight $\lambda$ and $U_{q}\left(\mathfrak{g}_{i}\right)$ is the subalgebra generated by Chevalley generators $e_{i}$ and $f_{i}$. If $M$ is integrable, we have

$$
M=\bigoplus_{0 \leq n \leq\left\langle h_{i}, \lambda\right\rangle} f_{i}^{(n)}\left(\operatorname{Ker} e_{i} \cap M_{\lambda}\right)
$$

Note that we use the following notations: $[m]_{i}=\left(q_{i}^{m}-q_{i}^{-m}\right) /\left(q_{i}-q_{i}^{-1}\right),[n]_{i} !=$ $\prod_{m=1}^{n}[m]_{i}, f_{i}^{(n)}=f_{i}^{n} /[n]_{i}$ ! with $q_{i}=q^{\left(\alpha_{i}, \alpha_{i}\right)}$, where $($,$) is an invariant bilinear$ form on $P$. We define the endomorphisms $\tilde{e}_{i}, \tilde{f}_{i}$ of $M$ by

$$
\tilde{f}_{i}\left(f_{i}^{(n)} u\right)=f_{i}^{(n+1)} u \quad \text { and } \quad \tilde{e}_{i}\left(f_{i}^{(n)} u\right)=f_{i}^{(n-1)} u
$$


for $u \in \operatorname{Ker} e_{i} \cap M_{\lambda}$ with $0 \leq n \leq\left\langle h_{i}, \lambda\right\rangle$. Similarly, we have

$$
M=\bigoplus_{0 \leq n \leq-\left\langle h_{i}, \mu\right\rangle} e_{i}^{(n)}\left(\operatorname{Ker} f_{i} \cap M_{\mu}\right) .
$$

These two decompositions are related as follows:

if $0 \leq n \leq\left\langle h_{i}, \lambda\right\rangle$ and $u \in \operatorname{Ker} e_{i} \cap M_{\lambda}$,

then $v=f_{i}^{\left(\left\langle h_{i}, \lambda\right\rangle\right)} u$ belongs to Ker $f_{i} \cap M_{s_{i}(\lambda)}$ and $f_{i}^{(n)} u=e_{i}^{\left(\left\langle h_{i}, \lambda\right\rangle-n\right)} v$.

Here $s_{i}(\lambda)=\lambda-\left\langle h_{i}, \lambda\right\rangle \alpha_{i}$. Hence we obtain

$$
\tilde{f}_{i}\left(e_{i}^{(n)} v\right)=e_{i}^{(n-1)} v \quad \text { and } \quad \tilde{e}_{i}\left(e_{i}^{(n)} v\right)=e_{i}^{(n+1)} v
$$

for $v \in \operatorname{Ker} f_{i} \cap M_{\mu}$ with $0 \leq n \leq-\left\langle h_{i}, \mu\right\rangle$.

Let us now look at the definition of a crystal base. Let $A$ be the subring of $\mathbb{Q}(q)$ consisting of rational functions without poles at $q=0$. Let $M$ be an integrable $U_{q}(\mathfrak{g})$-module.

Definition 2.1. A pair $(L, B)$ is called a crystal base of $M$ if it satisfies the following 6 conditions:

(2.5) $L$ is a free sub- $A$-module of $M$ such that $M \simeq \mathbb{Q}(q) \otimes_{A} L$,

(2.6) $B$ is a base of the $\mathbb{Q}$-vector space $L / q L$,

$$
\tilde{e}_{i} L \subset L \text { and } \tilde{f}_{i} L \subset L \text { for any } i \text {. }
$$

By (2.7) $\tilde{e}_{i}$ and $\tilde{f}_{i}$ act on $L / q L$.

$$
\begin{aligned}
& \tilde{e}_{i} B \subset B \cup\{0\} \text { and } \tilde{f}_{i} B \subset B \cup\{0\} . \\
& L=\oplus_{\lambda \in P} L_{\lambda} \text { and } B=\sqcup_{\lambda \in P} B_{\lambda}
\end{aligned}
$$

where $L_{\lambda}=L \cap M_{\lambda}$ and $B_{\lambda}=B \cap\left(L_{\lambda} / q L_{\lambda}\right)$.

$$
\text { For } b, b^{\prime} \in B, b^{\prime}=\tilde{f}_{i} b \text { if and only if } \tilde{e}_{i} b^{\prime}=b .
$$

Standard notations are in order. For $b \in B$ we set

(2.11) $\varepsilon_{i}(b)=\max \left\{m \in \mathbb{Z}_{\geq 0} \mid \tilde{e}_{i}^{m} b \neq 0\right\}$,

(2.12) $\varepsilon(b)=\sum_{i} \varepsilon_{i}(b) \Lambda_{i}$,

$$
\varphi_{i}(b)=\max \left\{m \in \mathbb{Z}_{\geq 0} \mid \tilde{f}_{i}^{m} b \neq 0\right\},
$$

(2.13) $\quad w t b=\varphi(b)-\varepsilon(b)$. 
Here $\left\{\Lambda_{i}\right\}$ stands for the set of fundamental weights of $\mathfrak{g}$.

The crystal base behaves nicely under the tensor product. Let $\left(L_{j}, B_{j}\right)$ be the crystal base of an integrable $U_{q}(\mathfrak{g})$-module $M_{j}(j=1,2)$. Set $L=L_{1} \otimes_{A} L_{2}$ and $B=\left\{b_{1} \otimes b_{2} \mid b_{j} \in B_{j}(j=1,2)\right\}$. Then $(L, B)$ is a crystal base of $M_{1} \otimes M_{2}$. Moreover, the action of $\tilde{e}_{i}$ and $\tilde{f}_{i}$ becomes very simple as

$$
\begin{aligned}
& \tilde{e}_{i}\left(b_{1} \otimes b_{2}\right)= \begin{cases}\tilde{e}_{i} b_{1} \otimes b_{2} & \text { if } \varphi_{i}\left(b_{1}\right) \geq \varepsilon_{i}\left(b_{2}\right), \\
b_{1} \otimes \tilde{e}_{i} b_{2} & \text { if } \varphi_{i}\left(b_{1}\right)<\varepsilon_{i}\left(b_{2}\right),\end{cases} \\
& \tilde{f}_{i}\left(b_{1} \otimes b_{2}\right)= \begin{cases}\tilde{f}_{i} b_{1} \otimes b_{2} & \text { if } \varphi_{i}\left(b_{1}\right)>\varepsilon_{i}\left(b_{2}\right), \\
b_{1} \otimes \tilde{f}_{i} b_{2} & \text { if } \varphi_{i}\left(b_{1}\right) \leq \varepsilon_{i}\left(b_{2}\right) .\end{cases}
\end{aligned}
$$

Here $0 \otimes b$ and $b \otimes 0$ are understood to be 0 . We denote this $B$ by $B_{1} \otimes B_{2}$. $\varepsilon_{i}, \varphi_{i}$ and $w t$ are given by

$$
\begin{aligned}
\varepsilon_{i}\left(b_{1} \otimes b_{2}\right) & =\max \left(\varepsilon_{i}\left(b_{1}\right), \varepsilon_{i}\left(b_{1}\right)+\varepsilon_{i}\left(b_{2}\right)-\varphi_{i}\left(b_{1}\right)\right), \\
\varphi_{i}\left(b_{1} \otimes b_{2}\right) & =\max \left(\varphi_{i}\left(b_{2}\right), \varphi_{i}\left(b_{1}\right)+\varphi_{i}\left(b_{2}\right)-\varepsilon_{i}\left(b_{2}\right)\right), \\
w t\left(b_{1} \otimes b_{2}\right) & =w t b_{1}+w t b_{2} .
\end{aligned}
$$

Next lemma is used later in Section 4.

Lemma 2.2. Let $(L, B)$ be a crystal base. Assume that $\tilde{e}_{i}^{3} b=\tilde{f}_{i}^{3} b=0$ for any $b \in B$. Let $v \in L$ be such that $v \equiv b \bmod q L$. Then we have

$$
\begin{aligned}
e_{i} v & \equiv q_{i}^{-\varphi_{i}(b)} \tilde{e}_{i} v \bmod q q_{i}^{-\varphi_{i}(b)} L, \\
f_{i} v & \equiv q_{i}^{-\varepsilon_{i}(b)} \tilde{f}_{i} v \bmod q q_{i}^{-\varepsilon_{i}(b)} L .
\end{aligned}
$$

In particular, $e_{i} v \equiv 0\left(\right.$ resp. $\left.f_{i} v \equiv 0\right)$ if $\varepsilon_{i}(b)=0\left(\right.$ resp. $\left.\varphi_{i}(b)=0\right)$.

Proof. We prove the second relation. Let $\lambda$ be the weight of $v$. From the assumption it suffices to check the relation for the following cases, since the other cases are trivial.

(i) $\varepsilon_{i}(b)=0,\left\langle h_{i}, \lambda\right\rangle=1$ or 2 ,

(ii) $\varepsilon_{i}(b)=0$ or $1,\left\langle h_{i}, \lambda\right\rangle=0$.

In case (i) we have $f_{i} v=\tilde{f}_{i} v$ by (2.1) and (2.2). In case (ii) let us write $v=$ $f_{i} v_{1}+v_{2}$ with $v_{j} \in \operatorname{Ker} e_{i} \cap L(j=1,2)$ such that $\left\langle h_{i}\right.$, wt $\left.v_{1}\right\rangle=2,\left\langle h_{i}\right.$, wt $\left.v_{2}\right\rangle=0$. Then we have $f_{i} v=f_{i}^{2} v_{1}=[2]_{i} \tilde{f}_{i} v \equiv q_{i}^{-1} \tilde{f}_{i} v \bmod q q_{i}^{-1} L$.

The first relation can be checked similarly by using (2.3) and (2.4). 


\section{§2.2. Polarization}

We define a total order on $\mathbb{Q}(q)$ by

$$
f>g \text { if and only if } f-g \in \bigsqcup_{n \in \mathbb{Z}}\left\{q^{n}(c+q A) \mid c>0\right\}
$$

and $f \geq g$ if $f>g$ or $f=g$.

Let $M$ and $N$ be $U_{q}(\mathfrak{g})$-modules. A bilinear form $():, M \otimes_{\mathbb{Q}(q)} N \rightarrow \mathbb{Q}(q)$ is called an admissible pairing if it satisfies

$$
\begin{aligned}
\left(q^{h} u, v\right) & =\left(u, q^{h} v\right), \\
\left(e_{i} u, v\right) & =\left(u, q_{i}^{-1} t_{i}^{-1} f_{i} v\right), \\
\left(f_{i} u, v\right) & =\left(u, q_{i}^{-1} t_{i} e_{i} v\right),
\end{aligned}
$$

for all $u \in M$ and $v \in N$. (2.19) implies

$$
\left(e_{i}^{(n)} u, v\right)=\left(u, q_{i}^{-n^{2}} t_{i}^{-n} f_{i}^{(n)} v\right), \quad\left(f_{i}^{(n)} u, v\right)=\left(u, q_{i}^{-n^{2}} t_{i}^{n} e_{i}^{(n)} v\right) .
$$

A symmetric bilinear form (, ) on $M$ is called a preporlarization of $M$ if it satisfies (2.19) for $u, v \in M$. A preporlarization is called a porlarization if it is positive definite with respective to the order on $\mathbb{Q}(q)$.

\section{$\S 2.3$. Fusion construction}

In what follows we assume that $\mathfrak{g}$ is of affine type. Let $P$ be the weight lattice, $\left\{\Lambda_{i}\right\}$ the set of fundamental weights and $\delta$ the generator of null roots of $\mathfrak{g}$. Then we have $P=\bigoplus_{i} \mathbb{Z} \Lambda_{i} \oplus \mathbb{Z} \delta$. We set

$$
P_{c l}=P / \mathbb{Z} \delta
$$

Similar to the quantum algebra $U_{q}(\mathfrak{g})$ which is associated with $P$, we can also consider $U_{q}^{\prime}(\mathfrak{g})$, which is associated with $P_{c l}$, namely, the subalgebra of $U_{q}(\mathfrak{g})$ generated by $e_{i}, f_{i}, q^{h}\left(h \in\left(P_{c l}\right)^{*}\right)$.

Let $K$ be a commutative ring containing $\mathbb{Q}(q)$ and let $x$ be an invertible element of $K$. We introduce a $K \otimes_{\mathbb{Q}(q)} U_{q}^{\prime}(\mathfrak{g})$-module $V_{x}$ by replacing the actions of $e_{i}, f_{i}$ with $x^{\delta_{i 0}} e_{i}, x^{-\delta_{i 0}} f_{i}$. The action of $q^{h}$ is not changed. Let $y$ also be an invertible element of $K$. A $K \otimes_{\mathbb{Q}(q)} U_{q}^{\prime}(\mathfrak{g})$-linear map

$$
R(x, y): V_{x} \otimes V_{y} \longrightarrow V_{y} \otimes V_{x}
$$


is called a $R$-matrix. Here we need to specify the coproduct $\Delta$ of $U_{q}(\mathfrak{g})$ we use in this paper. Our choice is the "lower" one (see [13]) given by

$$
\begin{aligned}
& \Delta\left(q^{h}\right)=q^{h} \otimes q^{h} \quad \text { for } h \in\left(P_{c l}\right)^{*}, \\
& \Delta\left(e_{i}\right)=e_{i} \otimes t_{i}^{-1}+1 \otimes e_{i}, \\
& \Delta\left(f_{i}\right)=f_{i} \otimes 1+t_{i} \otimes f_{i} .
\end{aligned}
$$

For a finite-dimensional $U_{q}^{\prime}(\mathfrak{g})$-module $V$ we assume the following.

(2.21) $V$ is irreducible.

(2.22) There exists $\lambda_{0} \in P_{c l}$ such that $w t V \subset \lambda_{0}+\sum_{i \neq 0} \mathbb{Z}_{\leq 0} \alpha_{i}$ and $\operatorname{dim} V_{\lambda_{0}}=1$.

Here $\left\{\alpha_{i}\right\}$ is the set of simple roots. Under these assumptions it is known that there exists a unique $R$-matrix up to a scalar multiple. Moreover, $R(x, y)$ depends only on $x / y$. Take a non zero vector $u_{0}$ from $V_{\lambda_{0}}$. We normalize $R(x, y)$ in such a way that $R(x, y)\left(u_{0} \otimes u_{0}\right)=u_{0} \otimes u_{0}$. It is known in [14] that if $V$ is a "good" module then the normalized $R$-matrix does not have a pole at $x / y=a \in A$.

Next we review the fusion construction following section 3 of [12]. Let $l$ be a positive integer and $\mathfrak{S}_{l}$ the $l$-th symmetric group. Let $s_{i}$ be the simple reflection which interchanges $i$ and $i+1$, and let $\ell(w)$ be the length of $w \in \mathfrak{S}_{l}$. Let $V$ be a finite-dimensional $U_{q}^{\prime}(\mathfrak{g})$-module. Let $R(x, y)$ denote the $R$-matrix for $V \otimes V$. For any $w \in \mathfrak{S}_{l}$ we construct a map $R_{w}\left(x_{1}, \ldots, x_{l}\right): V_{x_{1}} \otimes \cdots \otimes V_{x_{l}} \rightarrow$ $V_{x_{w(1)}} \otimes \cdots \otimes V_{x_{w(l)}}$ by

$$
\begin{aligned}
R_{1}\left(x_{1}, \ldots, x_{l}\right) & =1, \\
R_{s_{i}}\left(x_{1}, \ldots, x_{l}\right) & =\left(\bigotimes_{j<i} \operatorname{id}_{V_{x_{j}}}\right) \otimes R\left(x_{i}, x_{i+1}\right) \otimes\left(\bigotimes_{j>i+1} \operatorname{id}_{V_{x_{j}}}\right), \\
R_{w w^{\prime}}\left(x_{1}, \ldots, x_{l}\right) & =R_{w^{\prime}}\left(x_{w(1)}, \ldots, x_{w(l)}\right) \circ R_{w}\left(x_{1}, \ldots, x_{l}\right) \\
& \quad \text { for } w, w^{\prime} \text { such that } \ell\left(w w^{\prime}\right)=\ell(w)+\ell\left(w^{\prime}\right) .
\end{aligned}
$$

Fix $r \in \mathbb{Z}_{>0}$. For each $l \in \mathbb{Z}_{>0}$, we put

$$
\begin{aligned}
R_{l}= & R_{w_{0}}\left(q^{r(l-1)}, q^{r(l-3)}, \ldots, q^{-r(l-1)}\right): \\
& V_{q^{r(l-1)}} \otimes V_{q^{r(l-3)}} \otimes \cdots \otimes V_{q^{-r(l-1)}} \rightarrow V_{q^{-r(l-1)}} \otimes V_{q^{-r(l-3)}} \otimes \cdots \otimes V_{q^{r(l-1)}},
\end{aligned}
$$

where $w_{0}$ is the longest element of $\mathfrak{S}_{l}$. Then $R_{l}$ is a $U_{q}^{\prime}(\mathfrak{g})$-linear homomorphism. Define

$$
V_{l}=\operatorname{Im} R_{l}
$$


Let us denote by $W$ the image of

$$
R\left(q^{r}, q^{-r}\right): V_{q^{r}} \otimes V_{q^{-r}} \longrightarrow V_{q^{-r}} \otimes V_{q^{r}}
$$

and by $N$ its kernel. Then we have

$$
\begin{aligned}
& V_{l} \text { considered as a submodule of } V^{\otimes l}=V_{q^{-r(l-1)}} \otimes \cdots \otimes V_{q^{r(l-1)}} \\
& \text { is contained in } \bigcap_{i=0}^{l-2} V^{\otimes i} \otimes W \otimes V^{\otimes(l-2-i)} .
\end{aligned}
$$

Similarly, we have

$$
V_{l} \text { is a quotient of } V^{\otimes l} / \sum_{i=0}^{l-2} V^{\otimes i} \otimes N \otimes V^{\otimes(l-2-i)} \text {. }
$$

\section{§2.4. Preliminary propositions}

In this subsection, following [12] we define a prepolarization on $V_{l}$ and prepare a necessary proposition to show the main theorem. First we recall

Lemma 2.3. Let $M_{j}$ and $N_{j}$ be $U_{q}^{\prime}(\mathfrak{g})$-modules and let $(,)_{j}$ be an admissible pairing between $M_{j}$ and $N_{j}(j=1,2)$. Then the pairing (, ) between $M_{1} \otimes M_{2}$ and $N_{1} \otimes N_{2}$ defined by $\left(u_{1} \otimes u_{2}, v_{1} \otimes v_{2}\right)=\left(u_{1}, v_{1}\right)_{1}\left(u_{2}, v_{2}\right)_{2}$ for all $u_{j} \in M_{j}$ and $v_{j} \in N_{j}$ is admissible.

Let $V$ be a finite-dimensional $U_{q}^{\prime}(\mathfrak{g})$-module satisfying $(2.21),(2.22)$. Suppose $V$ has a polarization. The polarization on $V$ gives an admissible pairing between $V_{x}$ and $V_{x^{-1}}$. Hence it induces an admissible pairing between $V_{x_{1}} \otimes \cdots \otimes V_{x_{l}}$ and $V_{x_{1}^{-1}} \otimes \cdots \otimes V_{x_{l}^{-1}}$.

Lemma 2.4. If $x_{j}=x_{l+1-j}^{-1}$ for $j=1, \ldots, l$, then for any $u, u^{\prime} \in V_{x_{1}} \otimes$ $\cdots \otimes V_{x_{l}}$, we have

$$
\left(u, R_{w_{0}}\left(x_{1}, \ldots, x_{l}\right) u^{\prime}\right)=\left(u^{\prime}, R_{w_{0}}\left(x_{1}, \ldots, x_{l}\right) u\right) .
$$

By taking $x_{1}=q^{r(l-1)}, x_{2}=q^{r(l-3)}$, etc., we obtain the admissible pairing $(, \quad)$ between $W=V_{q^{r(l-1)}} \otimes V_{q^{r(l-3)}} \otimes \cdots \otimes V_{q^{-r(l-1)}}$ and $W^{\prime}=V_{q^{-r(l-1)}} \otimes$ $V_{q^{-r(l-3)}} \otimes \cdots \otimes V_{q^{r(l-1)}}$ that satisfies

$$
\left(w, R_{l} w^{\prime}\right)=\left(w^{\prime}, R_{l} w\right) \quad \text { for any } w, w^{\prime} \in W .
$$


This allows us to define a preporlarization $(,)_{l}$ on $V_{l}$ by

$$
\left(R_{l} u, R_{l} u^{\prime}\right)_{l}=\left(u, R_{l} u^{\prime}\right)
$$

for $u, u^{\prime} \in V_{q^{r(l-1)}} \otimes V_{q^{r(l-3)}} \otimes \cdots \otimes V_{q^{-r(l-1)}}$.

Next we introduce a $\mathbb{Z}$-form of $U_{q}^{\prime}(\mathfrak{g})$. Recall that $A$ is the subring of $\mathbb{Q}(q)$ consisting of rational functions without poles at $q=0$. We introduce the subalgebras $A_{\mathbb{Z}}$ and $K_{\mathbb{Z}}$ of $\mathbb{Q}(q)$ by

$$
\begin{aligned}
& A_{\mathbb{Z}}=\{f(q) / g(q) \mid f(q), g(q) \in \mathbb{Z}[q], g(0)=1\}, \\
& K_{\mathbb{Z}}=A_{\mathbb{Z}}\left[q^{-1}\right] .
\end{aligned}
$$

Then we have

$$
K_{\mathbb{Z}} \cap A=A_{\mathbb{Z}}, \quad A_{\mathbb{Z}} / q A_{\mathbb{Z}} \simeq \mathbb{Z} .
$$

We then define $U_{q}^{\prime}(\mathfrak{g})_{K_{\mathbb{Z}}}$ as the $K_{\mathbb{Z}}$-subalgebra of $U_{q}^{\prime}(\mathfrak{g})$ generated by $e_{i}, f_{i}, q^{h}$ $\left(h \in\left(P_{c l}\right)^{*}\right)$. Set $V_{K_{\mathbb{Z}}}=U_{q}^{\prime}(\mathfrak{g})_{K_{\mathbb{Z}}} u_{0}$ and assume

$$
\left(V_{K_{\mathbb{Z}}}\right)_{\lambda_{0}}=K_{\mathbb{Z}} u_{0}
$$

Let us further set

$$
\left(V_{l}\right)_{K_{\mathbb{Z}}}=R_{l}\left(\left(V_{K_{\mathbb{Z}}}\right)^{\otimes l}\right) \cap\left(V_{K_{\mathbb{Z}}}\right)^{\otimes l}
$$

Then one can show

Proposition 2.5. (i) $(,)_{l}$ is a nondegenerate prepolarization on $V_{l}$.

(ii) $\left(R_{l}\left(u_{0}^{\otimes l}\right), R_{l}\left(u_{0}^{\otimes l}\right)\right)_{l}=1$.

(iii) $\left(\left(V_{l}\right)_{K_{\mathbb{Z}}},\left(V_{l}\right)_{K_{\mathbb{Z}}}\right)_{l} \subset K_{\mathbb{Z}}$.

Let $I$ be the index set of the vertices of the Dynkin diagram of $\mathfrak{g}$ with the vertex 0 as in [10]. Let $\mathfrak{g}_{0}$ be the finite-dimensional simple Lie algebra whose Dynkin diagram is obtained by removing the 0 -vertex from that of $\mathfrak{g}$. Let $\bar{P}_{+}$be the set of dominant integral weights of $\mathfrak{g}_{0}$ and $V(\lambda)$ be the irreducible highest weight $U_{q}\left(\mathfrak{g}_{0}\right)$-module of highest weight $\lambda$ for $\lambda \in \bar{P}_{+}$. The following proposition, which is essentially stated in Proposition 2.6.1 and 2.6.2 of [12], is a key to prove the main theorem.

Proposition 2.6. Let $M$ be a finite-dimensional integrable $U_{q}^{\prime}(\mathfrak{g})$-module. Let $\left(\right.$, ) be a prepolarization on $M$, and $M_{K_{\mathbb{Z}}}$ a $U_{q}^{\prime}(\mathfrak{g})_{K_{\mathbb{Z}}}$-submodule of $M$ such that $\left(M_{K_{\mathbb{Z}}}, M_{K_{\mathbb{Z}}}\right) \subset K_{\mathbb{Z}}$. Let $\lambda_{1}, \ldots, \lambda_{m} \in \bar{P}_{+}$, and we assume the following conditions.

$$
\operatorname{dim} M_{\lambda_{k}} \leq \sum_{j=1}^{m} \operatorname{dim} V\left(\lambda_{j}\right)_{\lambda_{k}} \text { for } k=1, \ldots, m
$$




$$
\begin{aligned}
& \text { There exist } u_{j} \in\left(M_{K_{\mathbb{Z}}}\right)_{\lambda_{j}}(j=1, \ldots, m) \text { such that }\left(u_{j}, u_{k}\right) \in \delta_{j k}+ \\
& q A \text {, and }\left(e_{i} u_{j}, e_{i} u_{j}\right) \in q q_{i}^{-2\left(1+\left\langle h_{i}, \lambda_{j}\right\rangle\right)} \text { A for any } i \in I \backslash\{0\}
\end{aligned}
$$

Set $L=\{u \in M \mid(u, u) \in A\}$ and set $B=\left\{b \in M_{K_{\mathbb{Z}}} \cap L / M_{K_{\mathbb{Z}}} \cap q L \mid(b, b)_{0}=\right.$ $1\}$. Here $(,)_{0}$ is the $\mathbb{Q}$-valued symmetric bilinear form on $L / q L$ induced by (, ). Then we have the following.

(i) (, ) is a polarization on $M$.

(ii) $M \simeq \bigoplus_{j} V\left(\lambda_{j}\right)$ as $U_{q}\left(\mathfrak{g}_{0}\right)$-modules.

(iii) $(L, B)$ is a crystal pseudobase of $M$.

\section{§3. KR Module of Type $D$}

\section{§3.1. $\quad$ KR module $W_{1}^{(k)}$}

First we review the Dynkin datum of type $D_{n}^{(1)}$. Let $I=\{0,1, \ldots, n\}$ be the index set of the Dynkin diagram, $\left\{\alpha_{i}\right\}_{i \in I}$ the set of simple roots, $\left\{\Lambda_{i}\right\}_{i \in I}$ the set of fundamental weights. The standard null root $\delta$ is given by

$$
\delta=\alpha_{0}+\alpha_{1}+2 \alpha_{2}+\cdots+2 \alpha_{n-2}+\alpha_{n-1}+\alpha_{n} .
$$

We denote the weight lattice by $P$, that is, $P=\bigoplus_{i \in I} \mathbb{Z} \Lambda_{i} \oplus \mathbb{Z} \delta$. The sublattice $\bar{P}=\bigoplus_{i \in I_{0}} \mathbb{Z} \bar{\Lambda}_{i}$ can be viewed as the weight lattice for $D_{n}$. Here $I_{0}=I \backslash\{0\}$ and $\bar{\Lambda}_{i}=\Lambda_{i}-a_{i} \Lambda_{0}$ with $a_{i}$ being the coefficient of $\alpha_{i}$ in (3.1). It is sometimes useful to introduce an orthonormal basis $\left\{\epsilon_{1}, \epsilon_{2}, \ldots, \epsilon_{n}\right\}$ of $\mathbb{Q} \otimes_{\mathbb{Z}} \bar{P}$ in such a way that we have

$$
\begin{aligned}
\alpha_{i} & = \begin{cases}\epsilon_{i}-\epsilon_{i+1} & (i=1, \ldots, n-1) \\
\epsilon_{n-1}+\epsilon_{n} & (i=n),\end{cases} \\
\bar{\Lambda}_{i} & = \begin{cases}\epsilon_{1}+\cdots+\epsilon_{i} & (i=1, \ldots, n-2) \\
\left(\epsilon_{1}+\cdots+\epsilon_{n-1}-\epsilon_{n}\right) / 2 & (i=n-1) \\
\left(\epsilon_{1}+\cdots+\epsilon_{n-1}+\epsilon_{n}\right) / 2 & (i=n) .\end{cases}
\end{aligned}
$$

Then we have $\alpha_{0}=\delta-\epsilon_{1}-\epsilon_{2}$. Since the lengths of the simple roots are all equal, we have $q_{i}=q$ for any $i \in I$. Hence we shall abbreviate $i$ from $[m]_{i}$ or $[m]_{i} !$.

Let $W_{1}^{(k)}$ be the $k$-th fundamental representation of $U_{q}^{\prime}\left(D_{n}^{(1)}\right)$. It is known that it has the following decomposition into $U_{q}\left(D_{n}\right)$-modules.

$$
W_{1}^{(k)} \simeq \begin{cases}V\left(\bar{\Lambda}_{k}\right) \oplus V\left(\bar{\Lambda}_{k-2}\right) \oplus \cdots \oplus V\left(\bar{\Lambda}_{1} \text { or } 0\right) & \text { if } 1 \leq k \leq n-2 \\ V\left(\bar{\Lambda}_{k}\right) & \text { if } k=n-1, n\end{cases}
$$

On $W_{1}^{(k)}$ the following results are known. 
Proposition 3.1. (1) $W_{1}^{(k)}$ is "good" in the sense of Kashiwara. In particular, it has a crystal base.

(2) $W_{1}^{(k)}$ has a polarization.

The first claim is due to Kashiwara [14] and the second to Koga [18], who got the result by exploiting the fusion construction among the spin representations.

\section{§3.2. Crystal of $W_{1}^{(k)}$}

We denote the crystal of $W_{1}^{(k)}$ by $B^{k, 1}$. We review in this subsection Schilling's variation of Koga's result on the crystal structure of $B^{k, 1}$. First we treat the case of $k=1$. The crystal graph of $B^{1,1}$ is depicted as follows.

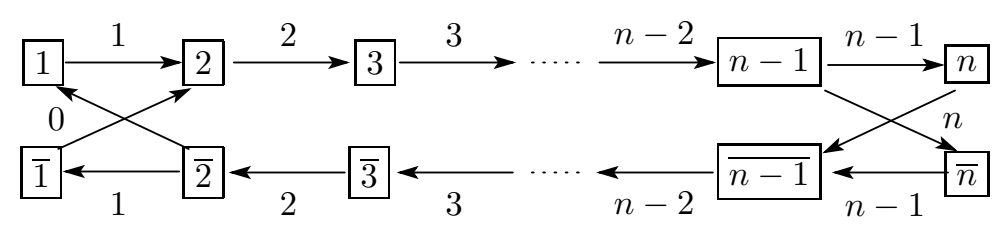

Here for $b, b^{\prime} \in B^{1,1} b \stackrel{i}{\longrightarrow}$ means $\tilde{f}_{i} b=b^{\prime}\left(\Leftrightarrow b=\tilde{e}_{i} b^{\prime}\right)$.

Next in view of (3.2) we recall the $U_{q}\left(D_{n}\right)$-crystal structure of $B\left(\bar{\Lambda}_{l}\right)$, the crystal of $U_{q}\left(D_{n}\right)$-module $V\left(\bar{\Lambda}_{l}\right)$, by [15]. Consider the alphabet $\mathcal{A}=$ $\{1,2, \ldots, n, \bar{n}, \overline{n-1}, \ldots, \overline{1}\}$ consisting of the crystal elements of $B^{1,1}$. It is given the following (partial) order.

$$
1 \prec 2 \prec \cdots \prec n-1 \prec \frac{n}{n} \prec \overline{n-1} \prec \cdots \prec \overline{1} .
$$

Then, for $1 \leq l \leq n-2 B\left(\bar{\Lambda}_{l}\right)$ is identified with the set of columns

$$
\begin{array}{|c|}
\hline m_{1} \\
\hline m_{2} \\
\hline \vdots \\
\hline m_{l} \\
\hline
\end{array}
$$

of height $l$ satisfying

$$
\begin{aligned}
& m_{j} \nsucc m_{j+1} \quad \text { for } j=1, \ldots, l-1, \\
& \text { if } m_{a}=p \text { and } m_{b}=\bar{p}, \text { then } \operatorname{dist}(p, \bar{p}) \leq p .
\end{aligned}
$$


Here $\operatorname{dist}(p, \bar{p})=a+l+1-b$ if $m_{a}=p$ and $m_{b}=\bar{p}$. The column tableau as above is also written as $m_{1} m_{2} \cdots m_{l}$. Note that we allow $\left(m_{j}, m_{j+1}\right)=(n, \bar{n})$ and $(\bar{n}, n)$. The actions of $\tilde{e}_{i}, \tilde{f}_{i}\left(i \in I_{0}\right)$ is given by considering the embedding

$$
\begin{gathered}
B\left(\bar{\Lambda}_{l}\right) \stackrel{\hookrightarrow}{\left(B^{1,1}\right)^{\otimes l}} \\
m_{1} m_{2} \cdots m_{l} \mapsto m_{1} \otimes m_{2} \otimes \cdots \otimes m_{l}
\end{gathered}
$$

and apply the tensor product rule of crystals on the r.h.s. $B(0)$ is realized as $\{\phi\}$ with the trivial actions of $\tilde{e}_{i}, \tilde{f}_{i}\left(i \in I_{0}\right)$, that is, $\tilde{e}_{i} \phi=\tilde{f}_{i} \phi=0$.

For $1 \leq k \leq n-2$, we are to represent $B^{k, 1}$ as the set of column tableaux of height $k$ satisfying (3.3). By (3.2) $B^{k, 1}$ is the union of the sets corresponding to $B\left(\bar{\Lambda}_{l}\right)$ with $0 \leq l \leq k$ and $l \equiv k(\bmod 2)$. In $[22]$ maps from $B\left(\bar{\Lambda}_{l}\right)$ to column tableaux of height $k$ were defined. If $b \in B\left(\bar{\Lambda}_{l}\right)$, then fill the column of height $l$ of $b$ succesively by a pair $\left(i_{j}, \overline{i_{j}}\right)$ for $1 \leq j \leq(k-l) / 2$ in the following way to obtain a column of height $k$. Set $i_{0}=0$. Let $i_{j-1}<i_{j}$ be minimal such that

(1) neither $i_{j}$ or $\overline{i_{j}}$ is in the column;

(2) adding $i_{j}$ and $\overline{i_{j}}$ to the column we have $\operatorname{dist}\left(i_{j}, \overline{i_{j}}\right) \geq i_{j}+j$;

(3) adding $i_{j}$ and $\overline{i_{j}}$ to the column, all other pairs $(a, \bar{a})$ in the new column with $a>i_{j}$ satisfy $\operatorname{dist}(a, \bar{a}) \leq a+j$.

The filling map and $\tilde{f}_{i}$ for $i \in I_{0}$ commute. Denote the filling map to height $k$ by $F_{k}$ or simply $F$. Let $D_{k}$ or $D$, the dropping map, be the inverse of $F_{k}$. Explicitly, given a one-column tableau of $b$ of height $k$, let $i_{0}=0$ and successively find $i_{j}>i_{j-1}$ minimal such that the pair $\left(i_{j}, \overline{i_{j}}\right)$ is in $b$ and $\operatorname{dist}\left(i_{j}, \overline{i_{j}}\right) \geq i_{j}+j$. Drop all such pairs $\left(i_{j}, \overline{i_{j}}\right)$ from $b$. Thus we have

$$
B^{k, 1} \simeq \bigoplus_{0 \leq l \leq k, l \equiv k(2)} F_{k}\left(B\left(\bar{\Lambda}_{l}\right)\right) \quad \text { as } U_{q}\left(D_{n}\right) \text {-crystals. }
$$

It is the set of all column tableaux of height $k$ satisfying (3.3) only.

We are left to give the rule of the actions of $\tilde{e}_{0}$ and $\tilde{f}_{0}$. For this purpose we need slight variants of $F_{k}$ and $D_{k}$, denoted by $\tilde{F}_{k}$ and $\tilde{D}_{k}$, respectively, which act on columns that do not contain $1,2, \overline{2}, \overline{1}$. On these columns $\tilde{F}_{k}$ and $\tilde{D}_{k}$ are defined by replacing $i \mapsto i-2$ and $\bar{i} \mapsto \overline{i-2}$, then applying $F_{k}$ and $D_{k}$, and finally replacing $i \mapsto i+2$ and $\bar{i} \mapsto \overline{i+2}$. The following proposition is given in $[22]$. 
Proposition 3.2. For $b \in B^{k, 1}$,

$$
\tilde{e}_{0} b= \begin{cases}F_{k}\left(\tilde{D}_{k-2}(x)\right) & \text { if } b=12 x \\ \tilde{F}_{k-1}(x) \overline{2} & \text { if } b=12 x \overline{2} \\ \tilde{F}_{k-1}(x) \overline{1} & \text { if } b=12 x \overline{1} \\ \tilde{F}_{k-2}(x) \overline{21} & \text { if } b=12 x \overline{21} \\ F_{k}\left(\tilde{D}_{k-1}(x) \overline{2}\right) & \text { if } b=1 x \\ F_{k}\left(\tilde{D}_{k-1}(x) \overline{1}\right) & \text { if } b=2 x \\ x \overline{21} & \text { if } b=1 x \overline{1} \text { and } \tilde{D}_{k-2}(x)=x \\ 0 & \text { otherwise }\end{cases}
$$

where $x$ does not contain $1,2, \overline{2}, \overline{1}$.

\section{§3.3. Existence of crystal pseudobase for $W_{l}^{(k)}$}

In this subsection we prove our main theorem by using Proposition 2.6. We prepare several lemmas and propositions.

Lemma 3.3. Let $R(x / y)$ be the R-matrix from $W_{1, x}^{(k)} \otimes W_{1, y}^{(k)}$ to $W_{1, y}^{(k)} \otimes$ $W_{1, x}^{(k)}$. Then it has the following form.

$$
R(z)=P_{2 \varpi_{k}}+\frac{z-q^{2}}{1-q^{2} z} P_{\varpi_{k+1}+\varpi_{k-1}}+\cdots .
$$

Here $z=x / y, \varpi_{j}=\epsilon_{1}+\epsilon_{2}+\cdots+\epsilon_{j} \in \bar{P}_{+}$for $0 \leq j \leq n-1$, and $P_{\lambda}$ stands for the projector onto the irreducible $U_{q}\left(D_{n}\right)$-module $V(\lambda)$ in $\left(W_{1}^{(k)}\right)^{\otimes 2}$.

Proof. Let $u_{0}$ be a $U_{q}\left(D_{n}\right)$-highest weight vector of $W_{1}^{(k)}$ of highest weight $\bar{\Lambda}_{k}\left(=\varpi_{k}\right)$. Since $V\left(\varpi_{k+1}+\varpi_{k-1}\right)$ is multiplicity free, a unique highest weight vector up to a scalar is given by

$$
v=u_{0} \otimes f_{k} u_{0}-q f_{k} u_{0} \otimes u_{0} .
$$


Since $f_{i} u_{0}=0$ for $i \in I \backslash\{k\}$, we have

$$
F^{(1)} v=u_{0} \otimes F^{(1)} u_{0}-q F^{(1)} u_{0} \otimes u_{0}
$$

where $F^{(1)}=f_{k+1} \cdots f_{n-2} f_{n} f_{n-1} \cdots f_{k+1} f_{1} \cdots f_{k-1}$. Hence we have

$$
F^{(2)} v=q u_{0} \otimes F^{(2)} u_{0}-q F^{(2)} u_{0} \otimes u_{0}+(\text { unwanted terms })
$$

where $F^{(2)}=f_{2} \cdots f_{k} F^{(1)}$ and we know $f_{0}$ (unwanted terms) $=0$ by weight consideration. Hence we have

$$
F^{(3)} v=q^{-1} y^{-1} u_{0} \otimes F^{(3)} u_{0}-q x^{-1} F^{(3)} u_{0} \otimes u_{0} \text { on } V_{x} \otimes V_{y}
$$

where $F^{(3)}=f_{0} F^{(2)}$ and $V=W_{1}^{(k)}$. Note that $F^{(3)} u_{0}=\alpha u_{0}$ with some $\alpha \neq 0$, since the corresponding crystal element is not killed from Proposition 3.2. Thus we have

$$
F^{(3)} v=\alpha\left(q^{-1} y^{-1}-q x^{-1}\right) u_{0} \otimes u_{0}
$$

Now let

$$
R(z) \propto \varphi(z) P_{2 \varpi_{k}}+\varphi^{\prime}(z) P_{\varpi_{k+1}+\varpi_{k-1}}+\cdots
$$

Then we have

$$
\begin{aligned}
R(z) F^{(3)} v & =\alpha\left(q^{-1} y^{-1}-q x^{-1}\right) \varphi(z)\left(u_{0} \otimes u_{0}\right) \\
=F^{(3)} R(z) v & =\varphi^{\prime}(z) F^{(3)} v^{\prime}=\alpha\left(q^{-1} x^{-1}-q y^{-1}\right) \varphi^{\prime}(z)\left(u_{0} \otimes u_{0}\right) .
\end{aligned}
$$

Here by $v^{\prime}$ we mean that it is considered to be in $V_{y} \otimes V_{x}$. Thus we have

$$
\varphi^{\prime}(z) / \varphi(z)=\frac{z-q^{2}}{1-q^{2} z} .
$$

Set $W=\operatorname{Im} R\left(q^{2}\right), N=\operatorname{Ker} R\left(q^{2}\right)$. They are $U_{q}^{\prime}\left(D_{n}^{(1)}\right)$-modules. Using the main result of $[20]$ one can show the following.

\section{Lemma 3.4. We have}

$$
W \simeq W_{2}^{(k)}, \quad N \simeq \bigotimes_{j \sim k} W_{1}^{(j)}
$$

as $U_{q}^{\prime}\left(D_{n}^{(1)}\right)$-modules. Here $j \sim k$ means that the corresponding vertices are tied by an edge in the Dynkin diagram. Moreover, both $W$ and $N$ are irreducible. 
Proof. In [20] it is shown that there exists an exact sequence of $U_{q}^{\prime}\left(D_{n}^{(1)}\right)$ modules

$$
0 \longrightarrow \bigotimes_{j \sim k} W_{1}^{(j)} \longrightarrow W_{1, q}^{(k)} \otimes W_{1, q^{-1}}^{(k)} \longrightarrow W_{2}^{(k)} \longrightarrow 0 .
$$

(An acute reader should have noticed that the exact sequence is different from [20]. It is because the definition of the KR modules and the choice of the coproduct are different.) Moreover, it is also known that $\bigotimes_{j \sim k} W_{1}^{(j)}$ and $W_{2}^{(k)}$ are irreducible. Set $W^{\prime}=\bigotimes_{j \sim k} W_{1}^{(j)}$ and consider $N \cap W^{\prime}$. Since $W^{\prime}$ is irreducible, we have $N \cap W^{\prime}=\{0\}$ or $W^{\prime}$. Recall that $W_{1, q}^{(k)} \otimes W_{1, q^{-1}}^{(k)}$ contains a unique irreducible $U_{q}\left(D_{n}\right)$-module $V\left(\varpi_{k+1}+\varpi_{k-1}\right)$. From the previous lemma and (3.2) we know it is contained both in $N$ and in $W^{\prime}$. Hence we have $N \supset W^{\prime}$. Now suppose $N \supsetneqq W^{\prime}$. Then we have a surjective $U_{q}^{\prime}\left(D_{n}^{(1)}\right)$-linear map

$$
W_{1, q}^{(k)} \otimes W_{1, q^{-1}}^{(k)} / W^{\prime} \longrightarrow W_{1, q}^{(k)} \otimes W_{1, q^{-1}}^{(k)} / N
$$

Since the l.h.s. is irreducible, $N=W^{\prime}$ or $W_{1, q}^{(k)} \otimes W_{1, q^{-1}}^{(k)}$. Since $N$ cannot be the second choice by the previous lemma. One obtains $N=W^{\prime}$ and $W \simeq W_{2}^{(k)}$.

Since $W$ is known to be a KR module by the previous lemma, we have

Lemma 3.5. As a $U_{q}\left(D_{n}\right)$-module $W$ has the following decomposition.

$$
W \simeq \bigoplus_{0 \leq m_{1} \leq m_{2} \leq[k / 2]} V\left(\bar{\Lambda}_{k-2 m_{1}}+\bar{\Lambda}_{k-2 m_{2}}\right)
$$

We set $B=B^{k, 1}$. We fix a basis $\left\{v_{I}\right\}_{I \in B}$ of $W_{1}^{(k)}$ in such a way that $v_{I} \bmod q L=I$ as an element of $B$.

Proposition 3.6. $\quad N$ contains a vector of the form

$$
v_{I_{1}} \otimes v_{I_{2}}-\sum_{J_{1} \otimes J_{2} \in B_{1}} a_{J_{1} J_{2}} v_{J_{1}} \otimes v_{J_{2}} \quad\left(a_{J_{1} J_{2}} \in A\right)
$$

for any $I_{1}, I_{2}$ such that $I_{1} \otimes I_{2} \in B^{\otimes 2} \backslash B_{1}$.

See (4.2),(4.4) for the definition of $B_{1}$.

We now apply the fusion construction in Section 2.3 to $V=W_{1}^{(k)}$ with $r=1$. The assumptions $(2.21),(2.22)$ are valid with $\lambda_{0}=\bar{\Lambda}_{k}$. (2.26) can also be checked. Other necessary properties are guaranteed by Proposition 3.1. For $l \in \mathbb{Z}_{>0}$ we define $W_{l}^{(k)}=\operatorname{Im} R_{l}$. Let $k^{\prime}=[k / 2]$. Let $\mathbf{c}=\left(c_{1}, c_{2}, \ldots, c_{k^{\prime}}\right)$ be 
a sequence of integers such that $l \geq c_{1} \geq c_{2} \geq \cdots \geq c_{k^{\prime}} \geq 0$. For such $\mathbf{c}$ we define a vector $u_{m}\left(0 \leq m \leq k^{\prime}\right)$ in $W_{l}^{(k)}$ inductively by

$$
u_{m}=\left(e_{k-2 m}^{\left(c_{m}\right)} \cdots e_{2}^{\left(c_{m}\right)} e_{1}^{\left(c_{m}\right)}\right)\left(e_{k-2 m+1}^{\left(c_{m}\right)} \cdots e_{3}^{\left(c_{m}\right)} e_{2}^{\left(c_{m}\right)}\right) e_{0}^{\left(c_{m}\right)} u_{m-1},
$$

where $u_{0}$ here is $u_{0}^{\otimes l}$ in $\left(W_{1}^{(k)}\right)^{\otimes l}$. Set $u(\mathbf{c})=u_{k^{\prime}}$. The weight of $u(\mathbf{c})$ is given by

$$
\lambda(\mathbf{c})=\sum_{j=0}^{k^{\prime}}\left(c_{j}-c_{j+1}\right) \bar{\Lambda}_{k-2 j},
$$

where we have set $c_{0}=l, c_{k^{\prime}+1}=0$. For $l, m \in \mathbb{Z}_{\geq 0}$ such that $m \leq l$ we define the $q$-binomial coefficient by

$$
\left[\begin{array}{c}
l \\
m
\end{array}\right]=\frac{[l] !}{[m] ![l-m] !} .
$$

The following proposition calculates values of prepolarizations necessary to prove the main theorem.

Proposition 3.7. (1) $(u(\mathbf{c}), u(\mathbf{c}))_{l}=\prod_{j=1}^{k^{\prime}} q^{c_{j}\left(2 l-c_{j}\right)}\left[\begin{array}{l}2 l \\ c_{j}\end{array}\right]$,

(2) $\left(e_{j} u(\mathbf{c}), e_{j} u(\mathbf{c})\right)_{l}=0$ unless $k-j \in 2 \mathbb{Z}_{\geq 0}$. If $k-j \in 2 \mathbb{Z}_{\geq 0}$, then setting $p=(k-j) / 2+1\left(e_{j} u(\mathbf{c}), e_{j} u(\mathbf{c})\right)_{l}$ is given by

$$
q^{2 l-c_{p-1}-1}\left[2 l-c_{p-1}\right] \prod_{j=1}^{k^{\prime}} q^{\left(c_{j}-\delta_{j, p}\right)\left(2 l-c_{j}\right)}\left[\begin{array}{l}
2 l-\delta_{j, p} \\
c_{j}-\delta_{j, p}
\end{array}\right] .
$$

Proofs of these propositions are given in subsequent sections.

The rest of this section is devoted to the proof of Theorem 1.1. From Proposition 2.6 it suffices to show

(i) $\operatorname{ch} W_{l}^{(k)} \leq \sum_{l \geq c_{1} \geq \cdots \geq c_{k^{\prime}} \geq 0} \operatorname{ch} V(\lambda(\mathbf{c}))$, where $V(\lambda)$ is the irreducible $U_{q}\left(D_{n}\right)$-module with highest weight $\lambda$ and $\operatorname{ch} V$ stands for the formal character of $V$.

(ii) $\left(u(\mathbf{c}), u\left(\mathbf{c}^{\prime}\right)\right)_{l} \in \delta_{\mathbf{c c}^{\prime}}+q A$ and $\left(e_{j} u(\mathbf{c}), e_{j} u(\mathbf{c})\right)_{l} \in q^{-1-2\left\langle h_{j}, \lambda(\mathbf{c})\right\rangle} A$ for $j \neq 0$.

Let us show (i). First notice that $\sum_{l \geq c_{1} \geq \cdots \geq c_{k^{\prime}} \geq 0} \operatorname{ch} V(\lambda(\mathbf{c}))=$ $\sum_{0 \leq m_{1} \leq \cdots \leq m_{l} \leq k^{\prime}} \operatorname{ch} V\left(\bar{\Lambda}_{k-2 m_{1}}+\cdots+\bar{\Lambda}_{k-2 m_{l}}\right)$. In view of (2.24) and Proposition $3.6 W_{l}^{(k)}$ is a quotient of a module generated by the set of vectors

$$
\left\{v_{I_{1}} \otimes v_{I_{2}} \otimes \cdots \otimes v_{I_{l}} \mid I_{j} \otimes I_{j+1} \in B_{1} \text { for } j=1, \ldots, l-1\right\} .
$$


Assume $I_{j} \in B\left(\bar{\Lambda}_{k-2 m_{l+1-j}}\right) \subset B$. Then $I_{1} \otimes I_{2} \otimes \cdots \otimes I_{l}$ belongs to

$$
\begin{aligned}
\bigcap_{j=1}^{l-1} B\left(\bar{\Lambda}_{k-2 m_{l}}\right) \otimes \cdots \otimes B\left(\bar{\Lambda}_{k-2 m_{j+2}}\right) & \otimes B\left(\bar{\Lambda}_{k-2 m_{j+1}}+\bar{\Lambda}_{k-2 m_{j}}\right) \\
& \otimes B\left(\bar{\Lambda}_{k-2 m_{j-1}}\right) \otimes \cdots \otimes B\left(\bar{\Lambda}_{k-2 m_{1}}\right) .
\end{aligned}
$$

However, the above crystal is known to be identified with $B\left(\bar{\Lambda}_{k-2 m_{1}}+\cdots+\right.$ $\bar{\Lambda}_{k-2 m_{l}}$ ) (see Proposition 2.2.1 of [15]). This fact verifies (i).

For the proof of (ii) note that

$$
[m] \in q^{1-m} A, \quad\left[\begin{array}{c}
m \\
n
\end{array}\right] \in q^{-n(m-n)} A .
$$

If $\mathbf{c} \neq \mathbf{c}^{\prime},\left(u(\mathbf{c}), u\left(\mathbf{c}^{\prime}\right)\right)_{l}=0$ since the weights of $u(\mathbf{c})$ and $u\left(\mathbf{c}^{\prime}\right)$ are different. $\left(u(\mathbf{c}), u\left(\mathbf{c}^{\prime}\right)\right)_{l} \in 1+q A$ by Proposition 3.7 (1). For the second part it suffices to notice that $\left\langle h_{i}, \lambda(\mathbf{c})\right\rangle \geq 0$. The proof is completed.

\section{$\S 4$. Proof of Proposition 3.6}

We prepare several lemmas. The next one is a direct consequence of Proposition 3.2 .

Lemma 4.1. Suppose $k \geq 2$. Set $k^{\prime}=[k / 2]$. For elements $b, b^{\prime}$ in $B^{k, 1}$ let $b \stackrel{\tilde{e}_{0}}{\longrightarrow} b^{\prime}$ mean $\tilde{e}_{0} b=b^{\prime}$. Then we have the following rules of 0 -actions. In (2)-(4) • stands for a crystal element whose explicit form is not used later.

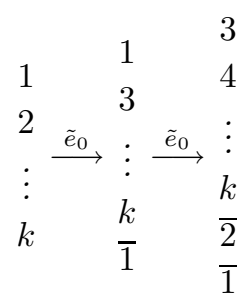


(2) Let $1 \leq m \leq k^{\prime}-1$.

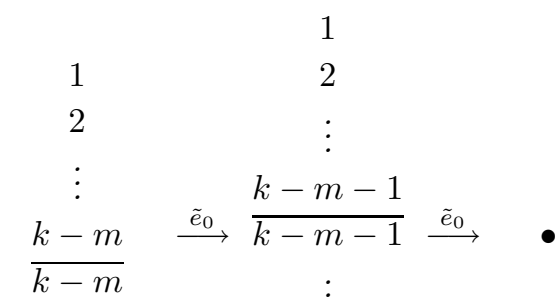

$$
\begin{aligned}
& \begin{array}{cc}
\frac{1}{k-2 m+1} & \overline{k-2 m+1} \\
\overline{2} & \overline{1}
\end{array}
\end{aligned}
$$

(3) Let $0 \leq m_{1} \leq m_{2} \leq k^{\prime}-1, m_{1} \leq p \leq \min \left(m_{21}, m_{s}-1\right)$, where $m_{21}=$ $m_{2}-m_{1}, m_{s}=m_{1}+m_{2}$.

$$
\begin{aligned}
& 1 \\
& 1 \\
& 2 \quad \text { : } \\
& \vdots \quad k-m_{21}-p-1 \\
& k-m_{21}-p \quad k-2 p+1 \\
& k-2 p+1 \\
& \begin{array}{cc}
\frac{k-2 m_{1}}{k-m_{21}-p} & k-2 m_{1} \\
& k-m_{21}-p-1
\end{array} \\
& \begin{array}{cc}
\frac{\vdots}{k-2 m_{2}+1} & \overline{k-2 m_{2}+1} \\
\overline{2} \\
\overline{1}
\end{array}
\end{aligned}
$$

(4) Let $1 \leq m_{1} \leq m_{2} \leq k^{\prime}-1, m_{21}+1 \leq p \leq m_{2}$, where $m_{21}=m_{2}-m_{1}$. Set $m_{s}=m_{1}+m_{2}$. 


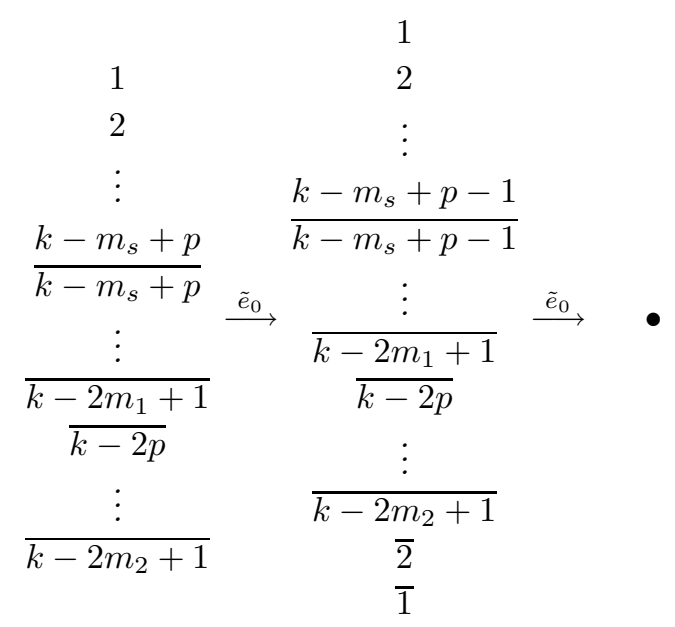

Lemma 4.2. $\quad$ Let $V$ be a $U_{q}\left(\mathfrak{g}_{0}\right)$-module with a crystal base $(L, B)$. Let $W$ a submodule of $V$. Let $\left\{b_{j} \mid j \in I\right\} \subset B$. Suppose $v$ is a vector in $W$ such that $v \equiv \sum_{j} b_{j} \bmod q L$. Decompose $I$ as $I=I_{1} \sqcup I_{2}$ by

$$
I_{1}=\left\{j \in I \mid \tilde{e}_{i} b_{j}=0 \text { for any } i\right\}, \quad I_{2}=\left\{j \in I \mid \tilde{e}_{i} b_{j} \neq 0 \text { for some } i\right\} .
$$

Then there exits a highest weight vector $w$ in $W$ such that $w \equiv \sum_{j \in I_{1}} b_{j} \bmod q L$.

Proof. By applying $\tilde{f}_{i} \tilde{e}_{i}$ we know that there exists a vector $v^{\prime}$ in $W$ such that $v^{\prime} \equiv \sum_{j \in I^{\prime}} b_{j} \bmod q L$, where $I^{\prime}=\left\{j \in I_{2} \mid \tilde{e}_{i} b_{j} \neq 0\right\}$. Hence there also exists a vector $v^{\prime \prime}$ in $W$ such that $v^{\prime \prime} \equiv \sum_{j \in\left(I^{\prime}\right)^{c}} b_{j} \bmod q L$. Continuing this with different $i$ 's, we obtain a vector $v^{\prime \prime \prime}$ in $W$ such that $v^{\prime \prime \prime} \equiv \sum_{j \in I_{1}} b_{j} \bmod q L$. Hence we can write $v^{\prime \prime \prime}$ as $v^{\prime \prime \prime}=w+w^{\prime}$ in such a way that $w \equiv \sum_{j \in I_{1}} b_{j} \bmod q L$ is a highest weight vector and $w^{\prime} \in q L$ is not, but we can remove $w^{\prime}$ from $v^{\prime \prime \prime}$.

In what follows in this section, by abuse of notation we represent a basis vector $v_{I}$ in $W_{1}^{(k)}$ also as $I$.

Lemma 4.3. Let $0 \leq m \leq k^{\prime}$. A highest weight vector of $V\left(2 \bar{\Lambda}_{k-2 m}\right)$ in $W$ is given by

$$
\begin{array}{cc}
1 & 1 \\
2 & 2 \\
\vdots & \vdots \\
\frac{k-m}{k-m} & \frac{k-m}{k-m} \\
\frac{\bmod q L .}{k-2 m+1} & \frac{\operatorname{mot}}{k-2 m+1}
\end{array}
$$


Proof. A highest weight vector of $V\left(2 \bar{\Lambda}_{k}\right)$ is given by

$$
\begin{array}{ccc}
1 & & 1 \\
2 & & 2 \\
\vdots & \otimes & \vdots \\
k & & k
\end{array}
$$

Noting that $W$ is a submodule of $W_{1, q^{-1}}^{(k)} \otimes W_{1, q}^{(k)}$, apply $e_{0}^{2}$ and use Lemma 2.2 and 4.1 (1). We obtain

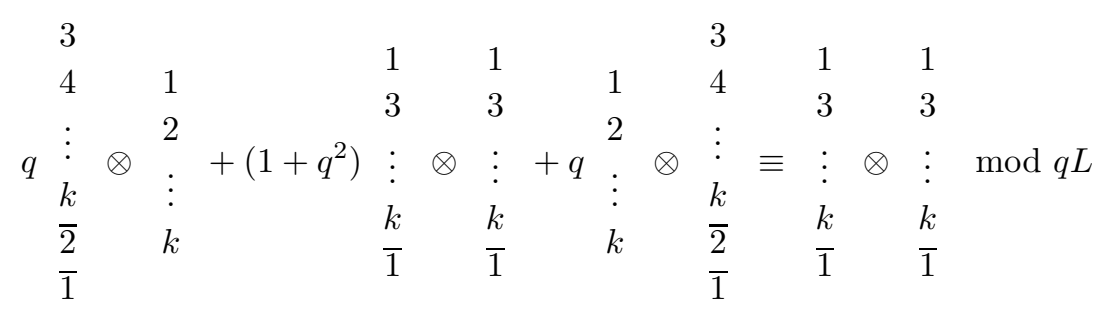

as a vector in $W$. Apply further $e_{k-2}^{(2)} \cdots e_{2}^{(2)} e_{1}^{(2)} e_{k-1}^{(2)} \cdots e_{3}^{(2)} e_{2}^{(2)}$, one obtains

$$
\begin{aligned}
& 1 \\
& 2 \quad 2 \\
& \vdots \otimes \bmod q L \\
& \frac{k-1}{k-1} \quad \frac{k-1}{k-1}
\end{aligned}
$$

as a vector in $W$.

For $m>1$, we prove by induction on $m$. By Lemma 2.2 and 4.1 (2), one has 


$$
\begin{aligned}
& 1 \\
& 2 \quad 2 \\
& e_{k-2 m-2}^{(2)} \cdots e_{2}^{(2)} e_{1}^{(2)} e_{k-2 m-1}^{(2)} \cdots e_{3}^{(2)} e_{2}^{(2)} e_{0}^{2} \frac{k-m}{k-m} \otimes \frac{k-m}{k-m} \\
& \frac{\vdots}{k-2 m+1} \quad \frac{\vdots}{k-2 m+1} \\
& 1 \\
& 2 \\
& \equiv \frac{k-m-1}{k-m-1} \otimes \frac{k-m-1}{k-m-1} \bmod q L, \\
& \frac{\vdots}{k-2 m-1} \frac{\vdots}{k-2 m-1}
\end{aligned}
$$

as a vector in $W$. Lemma 4.2 completes the proof.

Lemma 4.4. Let $0 \leq m_{1} \leq m_{2} \leq k^{\prime}$. Set $m_{21}=m_{2}-m_{1}, m_{s}=$ $m_{1}+m_{2}, M=\max \left(m_{1}, m_{21}\right)$. A highest weight vector of $V\left(\bar{\Lambda}_{k-2 m_{1}}+\bar{\Lambda}_{k-2 m_{2}}\right)$ in $W$ is given by

$$
\begin{aligned}
& 1 \text { 1 } \\
& \begin{array}{cccc} 
& 2 & & 2 \\
1 & \vdots & 1 & \vdots
\end{array}
\end{aligned}
$$

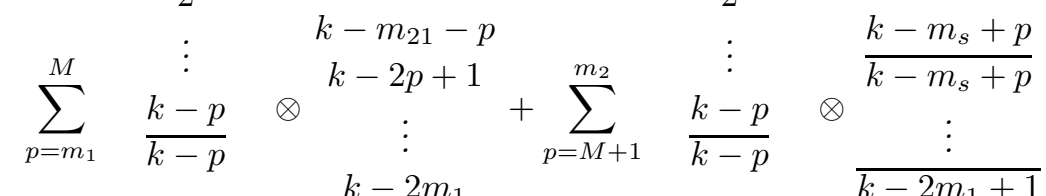

$$
\begin{aligned}
& \begin{array}{cccc}
\vdots & \frac{k-2 m_{1}}{k-m_{21}-p} & \vdots & \frac{k-2 m_{1}+1}{k-2 p} \\
\frac{k-2 p+1}{k-2 p+1} & .
\end{array} \\
& \overline{k-2 m_{2}+1} \quad \overline{k-2 m_{2}+1}
\end{aligned}
$$

$\bmod q L$.

Proof. We prove by induction on $m_{2}$. The case of $m_{2}=m_{1}$ is proved in the previous lemma. Assume $m_{1}>0$. Apply $e_{k-2 m_{2}-2} \cdots e_{1} e_{k-2 m_{2}-1} \cdots e_{2} e_{0}$ to (4.1) and use Lemma 2.2 and $4.1(2),(3),(4)$. Since one can always neglect 
terms corresponding to the crystal elements that are not killed by $\tilde{e}_{i}$ for some $i \neq 0$ by Lemma 4.2 , we obtain

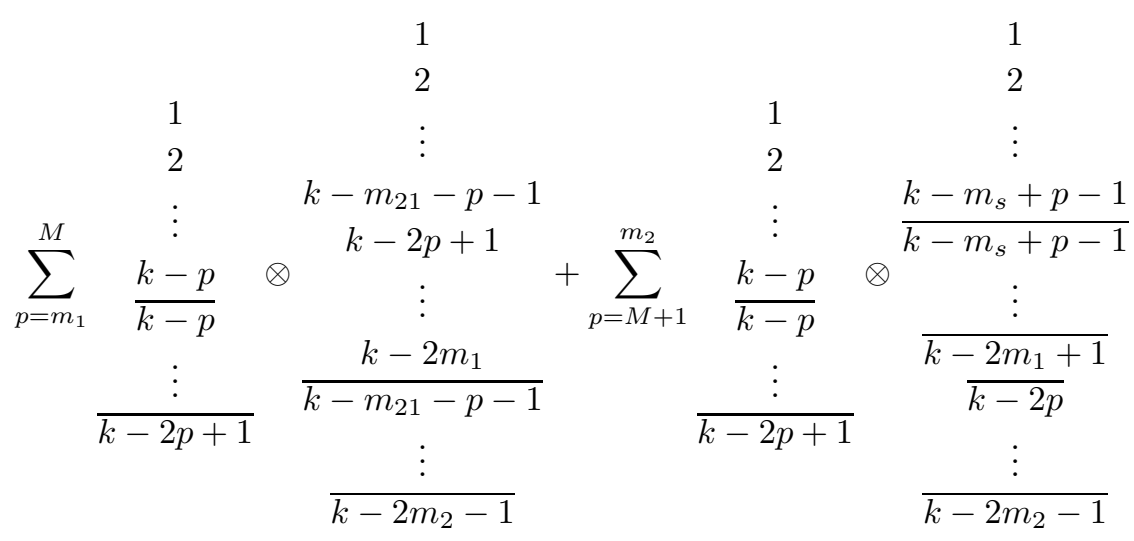

$$
\begin{aligned}
& 1 \quad 1 \\
& 2 \quad 2 \\
& +\frac{k-m_{2}-1}{k-m_{2}-1} \otimes \frac{k-m_{1}}{k-m_{1}} \\
& \frac{\vdots}{k-2 m_{2}-1} \quad \frac{\vdots}{k-2 m_{1}+1}
\end{aligned}
$$

$\bmod q L$ as a vector in $W$. Note that the last term can be regarded as the term in the summand of the middle term for $p=m_{2}+1$. If $m_{1}>m_{21}$, the induction proceeds. If $m_{1} \leq m_{21}$, note also that the term in the summand of the middle term for $p=M+1$ can be regarded as the one of the first term for $p=M+1$.

The proof for $m_{1}=0$ is similar, but needs some attention. Note that only the first summation survives in (4.1). Divide the cases into three: $p=0,1 \leq$ $p \leq m_{2}-1, p=m_{2}$ for the calculation of the action of $e_{0}$.

The following lemma is an easy consequence of (2.25) with $l=2$ and the nondegeneracy of the admissible pairing.

Lemma 4.5. Let $($,$) be the admissible pairing between W_{1, q}^{(k)} \otimes W_{1, q^{-1}}^{(k)}$ and $W_{1, q^{-1}}^{(k)} \otimes W_{1, q}^{(k)}$ induced from the poralization of $W_{1}^{(k)}$. Then $u \in N$ if and only if $(u, v)=0$ for any $v \in W$.

Now we are to prove Proposition 3.6. Set

$$
b\left(\bar{\Lambda}_{k-2 m}\right)=12 \cdots(k-m)(\overline{k-m}) \cdots(\overline{k-2 m+1}) \in B .
$$


$b\left(\bar{\Lambda}_{k-2 m}\right)$ is a highest weight vector of $V\left(\bar{\Lambda}_{k-2 m}\right)$ in $W_{1}^{(k)}$. We define the following subsets of $B^{\otimes 2}$.

$$
\begin{aligned}
& B_{1}^{h}=\left\{b\left(\bar{\Lambda}_{k-2 m_{2}}\right) \otimes b\left(\bar{\Lambda}_{k-2 m_{1}}\right) \mid 0 \leq m_{1} \leq m_{2} \leq k^{\prime}\right\}, \\
& B_{2}^{h}=\{\text { elements appearing in the summand of }(4.1)\} \backslash B_{1}^{h}, \\
& B_{a}=\left(\bigcup_{i_{1}, \ldots, i_{m} \in I_{0}} \tilde{f}_{i_{m}} \cdots \tilde{f}_{i_{1}} B_{a}^{h}\right) \backslash\{0\} \quad \text { for } a=1,2, \\
& B_{3}=B^{\otimes 2} \backslash\left(B_{1} \sqcup B_{2}\right), \quad B_{3}^{h}=\left\{b \in B_{3} \mid \tilde{e}_{i} b=0 \text { for any } i \neq 0\right\} .
\end{aligned}
$$

Note that (4.1) always contains $b\left(\bar{\Lambda}_{k-2 m_{2}}\right) \otimes b\left(\bar{\Lambda}_{k-2 m_{1}}\right)$. For $J=I_{1} \otimes I_{2} \in B^{\otimes 2}$ set $v_{J}=v_{I_{1}} \otimes v_{I_{2}}$.

Lemma 4.6. For any $J \in B_{2}^{h} \sqcup B_{3}^{h}$ there exists a highest weight vector $w_{J}$ in $N$ such that

$$
w_{J} \equiv \begin{cases}v_{J}-v_{J_{1}} & \text { if } J \in B_{2}^{h} \\ v_{J} & \text { if } J \in B_{3}^{h}\end{cases}
$$

$\bmod q L^{\otimes 2}$. Here $J_{1}$ is the unique element of $B_{1}^{h}$ that has the same weight as $J$.

Proof. If the weight of $J$ is not of the form of $\bar{\Lambda}_{k-2 m_{1}}+\bar{\Lambda}_{k-2 m_{2}}$, the assertion is clear. Suppose wt $J=\bar{\Lambda}_{k-2 m_{1}}+\bar{\Lambda}_{k-2 m_{2}}$. Consider a highest weight vector of the form

$$
v=c_{J_{1}} v_{J_{1}}+\sum_{J_{2}} c_{J_{2}} v_{J_{2}}+\sum_{J_{3}} c_{J_{3}} v_{J_{3}}+v^{\prime}
$$

Here $J_{1}$ is the unique element in $B_{1}^{h}$ of the fixed weight, $c_{J_{a}} \in A$ for $a=1,2,3$, the summation $\sum_{J_{a}}$ ranges over $J_{a} \in B_{a}^{h}$ that has the fixed weight for $a=2,3$, and $v^{\prime}$ is some vector in $q L^{\otimes 2}$. Let $w$ be the highest weight vector (4.1) in $W$. From Lemma $4.5 v \in N$ if and only if $(v, w)=0$. On the other hand, we have $(v, w) \equiv c_{J_{1}}+\sum_{J_{2}} c_{J_{2}} \bmod q A$ since $\left(L^{\otimes 2}, L^{\otimes 2}\right) \subset A$. Thus we have

$$
c_{J_{1}}+\sum_{J_{2}} c_{J_{2}} \equiv 0 \bmod q A .
$$

Hence for any $c_{J_{2}}, c_{J_{3}} \in A$, there exists a highest weight vector $v$ in $N$ such that $v \equiv \sum_{J_{2}} c_{J_{2}}\left(v_{J_{2}}-v_{J_{1}}\right)+\sum_{J_{3}} c_{J_{3}} v_{J_{3}} \bmod q L^{\otimes 2}$. The assertion follows from this by setting $c_{J}=1, c_{J^{\prime}}=0$ for $J^{\prime} \neq J$. 
Applying $\tilde{f}_{i}$ 's to $w_{J}$ for all $J$ in $B_{2}^{h} \sqcup B_{3}^{h}$ we obtain all weight vectors of $N$. Any such weight vector should have the following form

$$
a v_{J}+\sum_{J^{\prime} \neq J, J^{\prime} \in B_{2} \sqcup B_{3}} c_{J^{\prime}} v_{J^{\prime}}+\sum_{J^{\prime \prime} \in B_{1}} d_{J^{\prime \prime}} v_{J^{\prime \prime}},
$$

where $J \in B_{2} \sqcup B_{3}, a \in 1+q A, c_{J^{\prime}} \in q A, d_{J^{\prime \prime}} \in A$. By Gaussian elimination, we obtain the desired result.

\section{§5. $\quad$ Proof of Proposition 3.7}

We begin this section with an easy lemma.

Lemma 5.1. Let $v$ be a weight vector in $W_{l}^{(k)}$. If $\left(\right.$ wt $\left.v, \epsilon_{i}\right)>l$ for some $i \in\{1, \ldots, n\}$, then $v=0$.

Proof. The claim follows from the fact that $\left(w t u, \epsilon_{i}\right) \leq 1$ for a nonzero weight vector $u$ in $W_{1}^{(k)}$ and $W_{l}^{(k)}$ is a subspace of $W_{1}^{(k)}$.

The following formula will be used frequently.

$$
f_{i}^{(a)} e_{i}^{(b)}=\sum_{j=0}^{\min (a, b)} e_{i}^{(b-j)} f_{i}^{(a-j)}\left\{\begin{array}{c}
q^{a-b} t_{i}^{-1} \\
j
\end{array}\right\}
$$

where $\left\{\begin{array}{l}t \\ j\end{array}\right\}=\prod_{k=1}^{j}\left(q^{1-k} t-q^{k-1} t^{-1}\right) /\left(q^{k}-q^{-k}\right)$.

In this section we abbreviate $l$ of the preporlarization $(,)_{l}$ on $W_{l}^{(k)}$. We also write $|u|^{2}$ for $(u, u)$. Recall the definition of $u_{m}$ (3.5). wt $u_{m}$ is given by wt $u_{m}=\left(l-c_{1}\right) \bar{\Lambda}_{k}+\left(c_{1}-c_{2}\right) \bar{\Lambda}_{k-2}+\cdots+\left(c_{m-1}-c_{m}\right) \bar{\Lambda}_{k-2 m+2}+c_{m} \bar{\Lambda}_{k-2 m}$.

\section{Lemma 5.2.}

$$
\left|u_{m}\right|^{2}=q^{c_{m}\left(2 l-c_{m}\right)}\left[\begin{array}{c}
2 l \\
c_{m}
\end{array}\right]\left|u_{m-1}\right|^{2} .
$$

Proof. Since the other case is similar, we prove when $k$ is even. Using (2.20), we have

$$
\left|u_{m}\right|^{2}=\left(\left(e_{k-2 m-1}^{\left(c_{m}\right)} \cdots e_{1}^{\left(c_{m}\right)}\right)\left(e_{k-2 m+1}^{\left(c_{m}\right)} \cdots e_{2}^{\left(c_{m}\right)}\right) e_{0}^{\left(c_{m}\right)} u_{m-1}, f_{k-2 m}^{\left(c_{m}\right)} u_{m}\right) .
$$

By (5.1) we obtain

(5.2) $f_{k-2 m}^{\left(c_{m}\right)} u_{m}$

$$
=\sum_{j} e_{k-2 m}^{\left(c_{m}-j\right)} f_{k-2 m}^{\left(c_{m}-j\right)}\left[\begin{array}{c}
c_{m} \\
j
\end{array}\right]\left(e_{k-2 m-1}^{\left(c_{m}\right)} \cdots e_{1}^{\left(c_{m}\right)}\right)\left(e_{k-2 m+1}^{\left(c_{m}\right)} \cdots e_{2}^{\left(c_{m}\right)}\right) e_{0}^{\left(c_{m}\right)} u_{m-1} .
$$


Note that wt $f_{k-2 m}^{\left(c_{m}-j\right)}\left(e_{k-2 m-1}^{\left(c_{m}\right)} \cdots e_{1}^{\left(c_{m}\right)}\right)\left(e_{k-2 m+1}^{\left(c_{m}\right)} \cdots e_{2}^{\left(c_{m}\right)}\right) e_{0}^{\left(c_{m}\right)} u_{m-1}=w t u_{m}-$ $\left(2 c_{m}-j\right) \alpha_{k-2 m}$. From Lemma 5.1 the summand in the r.h.s. of (5.2) becomes 0 unless $j=c_{m}$. Hence we have

$$
\left|u_{m}\right|^{2}=\left|\left(e_{k-2 m-1}^{\left(c_{m}\right)} \cdots e_{1}^{\left(c_{m}\right)}\right)\left(e_{k-2 m+1}^{\left(c_{m}\right)} \cdots e_{2}^{\left(c_{m}\right)}\right) e_{0}^{\left(c_{m}\right)} u_{m-1}\right|^{2} .
$$

Similar calculations continue until we arrive at $\left|u_{m}\right|^{2}=\left|e_{0}^{\left(c_{m}\right)} u_{m-1}\right|^{2}$. Using (2.20),(5.1) and Lemma 5.1 again, we this time have $\left|u_{m}\right|^{2}=$ $q^{c_{m}\left(2 l-c_{m}\right)}\left[\begin{array}{c}2 l \\ c_{m}\end{array}\right]\left|u_{m-1}\right|^{2}$.

Lemma 5.3. (1) $e_{j} u_{k^{\prime}}=0$ when $k$ is even, if $j \geq k+1$ or $j=1$ when $k$ is $o d d$.

(2) $\left|f_{j} u_{p}\right|^{2}=q^{c_{p}\left(2 l-1-c_{p}\right)}\left[\begin{array}{c}2 l-1 \\ c_{p}\end{array}\right] q^{c_{p-1}-1}\left[c_{p-1}\right]\left|u_{p}\right|^{2}$ if $j=k-2 p+2, p=$ $1, \ldots, k^{\prime}$.

(3) $\left|f_{j} u_{p}\right|^{2}=0$ if $j=k-2 p+1, p=1, \ldots, k^{\prime}$.

Proof. (1) Write $u_{k^{\prime}}=E u_{0}$. If $j \geq k+1, e_{j}$ commutes with $E$. The claim follows from $e_{j} u_{0}=0$. When $k$ is odd, $e_{1} u_{k^{\prime}}=0$ follows from Lemma 5.1 .

(2) When $c_{p}=0$, the equality is shown as follows.

$$
\begin{aligned}
\left|f_{j} u_{p}\right|^{2} & =\left|f_{k-2 p+2} u_{p-1}\right|^{2} \\
& =\left(u_{p-1}, q^{-1} t_{k-2 p+2} e_{k-2 p+2} f_{k-2 p+2} u_{p-1}\right) \\
& =q^{c_{p-1}-1}\left[c_{p-1}\right]\left|u_{p-1}\right|^{2} .
\end{aligned}
$$

Here we have used the relation $e_{k-2 p+2} u_{p-1}=0$, that can be confirmed by Lemma 5.1.

Now assume $c_{p}>0$. Imitating the proof of Lemma 5.2, one obtains

$$
\left|f_{j} u_{p}\right|^{2}=\left|\left(e_{k-2 p+1}^{\left(c_{p}\right)} \cdots e_{2}^{\left(c_{p}\right)}\right) e_{0}^{\left(c_{p}\right)} \cdot f_{f-2 p+2} u_{p-1}\right|^{2} .
$$

Next we calculate

$$
\begin{aligned}
& q^{-c_{p}^{2}} t_{k-2 p+1}^{-c_{p}} f_{k-2 p+1}^{\left(c_{p}\right)}\left(e_{k-2 p+1}^{\left(c_{p}\right)} \cdots e_{2}^{\left(c_{p}\right)}\right) e_{0}^{\left(c_{p}\right)} \cdot f_{k-2 p+2} u_{p-1} \\
& =q^{-c_{p}} \sum_{j} e_{k-2 p+1}^{\left(c_{p}-j\right)} f_{k-2 p+1}^{\left(c_{p}-j\right)}\left[\begin{array}{c}
c_{p}-1 \\
j
\end{array}\right] e_{k-2 p}^{\left(c_{p}\right)} \cdots e_{2}^{\left(c_{p}\right)} e_{0}^{\left(c_{p}\right)} \cdot f_{k-2 p+2} u_{p-1} .
\end{aligned}
$$

Since $\left(w t f_{k-2 p+1}^{\left(c_{p}-j\right)} e_{k-2 p}^{\left(c_{p}\right)} \cdots e_{2}^{\left(c_{p}\right)} e_{0}^{\left(c_{p}\right)} f_{k-2 p+2} u_{p-1}, \epsilon_{k-2 p+2}\right)=l-1+c_{p}-j$, the summand of the above expression survives only when $j=c_{p}, c_{p}-1$. Noting $\left[\begin{array}{c}c_{p}-1 \\ c_{p}\end{array}\right]=0$, we obtain

$$
\left|f_{j} u_{p}\right|^{2}=\left|\left(e_{k-2 p}^{\left(c_{p}\right)} \cdots e_{2}^{\left(c_{p}\right)}\right) e_{0}^{\left(c_{p}\right)} \cdot f_{k-2 p+1} f_{k-2 p+2} u_{p-1}\right|^{2} .
$$


Calculating similarly, one gets $\left|f_{j} u_{p-1}\right|^{2}=\left|e_{0}^{\left(c_{p}\right)} \cdot f_{2} \cdots f_{k-2 p+2} u_{p-1}\right|^{2}$. After removing $e_{0}^{\left(c_{p}\right)}$, we arrive at

$$
\left|f_{j} u_{p}\right|^{2}=q^{c_{p}\left(2 l-1-c_{p}\right)}\left[\begin{array}{c}
2 l-1 \\
c_{p}
\end{array}\right]\left|f_{2} \cdots f_{k-2 p+2} u_{p-1}\right|^{2} .
$$

Calculating similarly, we obtain

$$
\left|f_{2} \cdots f_{k-2 p+2} u_{p-1}\right|^{2}=\left|f_{k-2 p+2} u_{p-1}\right|^{2}=q^{c_{p-1}-1}\left[c_{p-1}\right]\left|u_{p-1}\right|^{2} .
$$

(3) The proof goes parallel to that of (2). When $c_{p}=0$,

$$
\left|f_{j} u_{p}\right|^{2}=\left|f_{k-2 p+1} u_{p-1}\right|^{2}=0
$$

from Lemma 5.1. Assume $c_{p}>0$. One obtains

$$
\left|f_{j} u_{p}\right|^{2}=\left|f_{1} f_{2} \cdots f_{k-2 p+1} u_{p-1 / 2}\right|^{2} .
$$

Noting that $f_{i} u_{p-1}=0$ for $i=1, \ldots, k-2 p+1$, we have

$$
\begin{aligned}
f_{1} f_{2} \cdots f_{k-2 p+1} u_{p-1 / 2} & =f_{1} f_{2} \cdots f_{k-2 p+1}\left(e_{k-2 p+1}^{\left(c_{p}\right)} \cdots e_{2}^{\left(c_{p}\right)}\right) e_{0}^{\left(c_{p}\right)} u_{p-1} \\
& =\alpha \cdot f_{1}\left(e_{k-2 p+1}^{\left(c_{p}-1\right)} \cdots e_{2}^{\left(c_{p}-1\right)}\right) \cdot e_{0}^{\left(c_{p}\right)} u_{p-1}=0 .
\end{aligned}
$$

Here $\alpha$ is a product of $q$-integers.

The proof is complete.

Now we are in a position to prove Proposition 3.7. (1) is a simple consequence of Lemma 5.2. (2) when $j \geq k+1$ is settled by Lemma 5.3 (1). To show when $j \leq k$ note that

$$
\begin{aligned}
\left|e_{j} u_{k^{\prime}}\right|^{2} & =\left(u_{k^{\prime}}, q^{-1} t_{j}^{-1} f_{j} e_{j} u_{k^{\prime}}\right) \\
& =q^{2 \beta_{j}}\left|f_{j} u_{k^{\prime}}\right|^{2}+q^{\beta_{j}-1}\left[\beta_{j}\right]\left|u_{k^{\prime}}\right|^{2},
\end{aligned}
$$

where

$$
\beta_{j}=-\left\langle h_{j}, w t u_{k^{\prime}}\right\rangle= \begin{cases}c_{\frac{k-j}{2}+1}-c_{\frac{k-j}{2}} & \text { if } j \equiv k(2), \\ 0 & \text { if } j \neq k(2) .\end{cases}
$$

Thus we are left to evaluate $\left|f_{j} u_{k^{\prime}}\right|^{2}$. Examining the proof of Lemma 5.2 carefully, one notices that the same recursion formula is valid when $m>p$, namely, one has

$$
\left|f_{j} u_{m}\right|^{2}=q^{c_{m}\left(2 l-c_{m}\right)}\left[\begin{array}{c}
2 l \\
c_{m}
\end{array}\right]\left|f_{j} u_{m-1}\right|^{2} \quad \text { for } m>p .
$$

The formula for $\left|f_{j} u_{k^{\prime}}\right|^{2}$ is obtained from this, Lemma 5.3 (2) or (3) and Lemma 5.2. Calculating explicitly we obtain (2). 


\section{Acknowledgments}

The author thanks Masaki Kashiwara, Hiraku Nakajima, Satoshi Naito and Daisuke Sagaki for stimulating discussions. He is partially supported by Grant-in-Aid for Scientific Research (C) 18540030, Japan Society for the Promotion of Science.

\section{References}

[1] J. Beck and H. Nakajima, Crystal bases and two-sided cells of quantum affine algebras, Duke Math. 123 (2004), 335-402.

[2] G. Benkart, I. Frenkel, S-J. Kang and H. Lee, Level 1 perfect crystals and path realizations of basic representations at $q=0$, Internat. Math. Res. Notices 2006, Art. ID 10312, 28 pp.

[3] V. Chari, On the fermionic formula and the Kirillov-Reshetikhin conjecture, Internat. Math. Res. Notices 2001, no. 12, 629-654.

[4] V. Chari and A. Pressley, Quantum affine algebras and their representations, in Representations of groups, (Banff, AB, 1994), 59-78, Amer. Math. Soc., Providence, RI.

[5] V. G. Drinfel'd, A new realization of Yangians and quantized affine algebras, Dokl. Akad. Nauk SSSR 296 (1987), no. 1, 13-17; translation in Soviet Math. Dokl. 36 (1988), no. 2, 212-216.

[6] G. Hatayama, A. Kuniba, M. Okado, T. Takagi and Z. Tsuboi, Paths, crystals and fermionic formulae, in MathPhys Odyssey 2001, 205-272, Birkhäuser, Boston, Boston, MA.

[7] G. Hatayama, A. Kuniba, M. Okado, T. Takagi, Y. Yamada, Remarks on fermionic formula, in Resent developments in quantum affine algebras and related topics (Raleigh, NC, 1998) 243-291 Contemp. Math. 248, Amer. Math. Soc., Providence, RI.

[8] D. Hernandez, The Kirillov-Reshetikhin conjecture and solution of $T$-systems, J. Reine Angew. Math. 596 (2006), 63-87.

[9] N. Jing, K. C. Misra and M. Okado, $q$-wedge modules for quantized enveloping algebras of classical type, J. Algebra. 230 (2000), 518-539.

[10] V. G. Kac, "Infinite Dimensional Lie Algebras," 3rd ed., Cambridge Univ. Press, Cambridge, UK, 1990.

[11] S-J. Kang, M. Kashiwara, K. C. Misra, Crystal bases of Verma modules for quantum affine Lie algebras, Compositio Math. 92 (1994), 299-325.

[12] S-J. Kang, M. Kashiwara, K. C. Misra, T. Miwa, T. Nakashima and A. Nakayashiki, Perfect crystals of quantum affine Lie algebras, Duke Math. J. 68 (1992) 499-607.

[13] M. Kashiwara, On crystal bases of the $q$-analogue of universal enveloping algebras, Duke Math. J. 63 (1991), 465-516.

[14] - On level zero representations of quantized affine algebras, Duke Math. J. 112 (2002), 117-175.

[15] M. Kashiwara and T. Nakashima, Crystal graphs for representations of $q$-analogue of classical Lie algebras, J. Algebra 163 (1994), 295-345.

[16] A. N. Kirillov, Completeness of states of the generalized Heisenberg magnet, J. Sov. Math. 36 (1987) 115-128.

[17] A. N. Kirillov and N. Yu. Reshetikhin, Representations of Yangians and multiplicity of occurrence of the irreducible components of the tensor product of representations of simple Lie algebras, J. Sov. Math. 52 (1990), 3156-3164. 
[18] Y. Koga, Level one perfect crystals for $B_{n}^{(1)}, C_{n}^{(1)}$ and $D_{n}^{(1)}$, J. Algebra 217 (1999), 312-334.

[19] S. Naito and D. Sagaki, Path model for a level-zero extremal weight module over a quantum affine algebra, Int. Math. Res. Not. 2003, no. 32, 1731-1754.

[20] H. Nakajima, $t$-analogues of $q$-characters of Kirillov-Reshetikhin modules of quantum affine algebras, Represent. Theory 7 (2003), 259-274.

[21] Extremal weight modules of quantum affine algebras, in Representation theorg of algebraic groups and quantum groups, 343-369 Adv. Stud. Pure Math. 40, Math. Soc. Japan. Tokyo.

[22] A. Schilling, A bijection between type $D_{n}^{(1)}$ crystals and rigged configurations, J. Algebra 285 (2005), 292-334.

[23] P. Sternberg and A. Schilling, Finite-dimensional crystals $B^{2, s}$ for quantum affine algebras of type $D_{n}^{(1)}$, J. Algebraic. Combin. 23 (2006), 317-354.

[24] M. Varagnolo and E. Vasserot, Canonical bases and quiver varieties, Represent. Theory 7 (2003), 227-258.

[25] S. Yamane, Perfect crystals of $U_{q}\left(G_{2}^{(1)}\right)$, J. Algebra 210 (1998), 440-486. 